\title{
Drinking Juice and Childhood Obesity
}

\author{
Zohreh Shoar, M.D., M.P.H.
}

The mother of my 12 month old patient looked surprised at me when I told her that she should avoid giving juice to her overweight child. Instead, she asked me a legitimate question: "Then why does the WIC give juice to us?"

It took me a while to come up with an answer and then I just simply said: "It is wrong and they should not provide juice through their program". She smiled and I tried to explain to her about the effect of juice, as a pure sugary drink, on dental and oral health and emphasized healthy eating habits to prevent overweight and obesity.

I am not sure if I have been successful in convincing her, but here is my question:

It is well known that childhood obesity is associated with increased mortality and morbidity during childhood and later as an adult. Accordingly, with escalating epidemics of childhood obesity in recent years, the prevalence of its resultant co-morbidities such as metabolic syndrome, type 2 diabetes, and cardio-vascular diseases are dramatically increasing. Based on Center for Disease Control and Prevention (CDC) report, currently 17 percent of children ages 2-19 years are obese and in spite of multiple programs and initiatives, including first lady Michelle Obama's "Let's Move", childhood obesity rates continue to remain high and unchanged since the last CDC report on 2003-2004. Similarly, a recent study has shown that the life expectancy for overweight and obese adolescents during the last 40 years has remained stable, while it has significantly dropped in normal weight children. Wherefore, looking at the programs like Special Supplemental Nutrition Program for Women, Infants, and Children (WIC) that mainly focuses on the nutritional needs of low-income families is a necessity. While overweight/obesity seems to be everybody's business, shouldn't we start from ourselves? 